Rapp. Grønlands geol. Unders. 73, 81-85 (1976)

\title{
FIELD RELATIONSHIPS AND PETROLOGY OF LEUCOCRATIC SCHISTS FROM THE RAVNS STOR $\varnothing$ GROUP NEAR FISKENÆSSET
}

\author{
Clark R. L. Friend
}

\begin{abstract}
Introduction
During field work in 1971-72 whilst mapping the Ravns Storø amphibolite belt (Andersen \& Friend, 1973) a series of leucocratic schists were found. These occur along the south-east side of the belt on the islands fkatup nunâ, Sarqarigsup nunâ, Qagssikasaup nunâ and Ikerasatsiaup nunâ.

These rocks are initially striking because of their colour, pale cream or yellowish, with the frequent development of spectacular garbenschiefer textures (Spry, 1969) in pale brown, honey or colourless amphiboles (fig. 41).
\end{abstract}

\section{Field relations}

The schists are found towards the base of the regionally metamorphosed extrusive Qeqertarssuaq formation of the Ravns Storø group. Their exact position is not known since the true base of the formation has been removed by migmatisation and deformation. They occur in a unit of finely interlayered meta-pillow lavas, garnet amphibolites, black or dark green schistose to massive amphibolites and minor meta-sedimentary rocks. The unit thickens south-westwards, with the leucocratic schists also thickening but becoming relatively less important members. These rocks appear to continue laterally to Qeqertarssuaq to the north-east, as a staurolite-biotite-muscovite-chlorite-plagioclase-quartz rock (L. S. Andersen, personal communication). They are associated on Íkátup nunâ with three units of biotite-garnet-staurolite-hornblende schist believed to be of sedimentary origin (Table 12). These layers rarely exceed $50 \mathrm{~cm}$ in thickness and frequently are pinched out forming irregular boudins. The unit is remarkable for the development of rhombdodecahedral garnet porphyroblasts up to $11 \mathrm{~cm}$ in diameter (Giesecke, 1910; Dawes, 1970).

The leucocratic schists occur as distinct horizons amongst the amphibolites with no observed transitions. Banding is evident in some which reflects either original compositional layering or may be due to metamorphic segregation. Since there are no observed cross cutting relationships along their boundaries they are believed to be an initial part of the sequence and not later intrusives.

A study of their chemistry indicates that they are not originally sediments since they have high nickel values. A tuffaceous origin has been considered, as this is favoured for the production of garbenschiefer textures (Spry, 1969), leaving the mineral assemblages as a function of their chemistry. 
Table 12. Average analysis of biotite-garnet-staurolite hornblende schist of Ravns Storø group

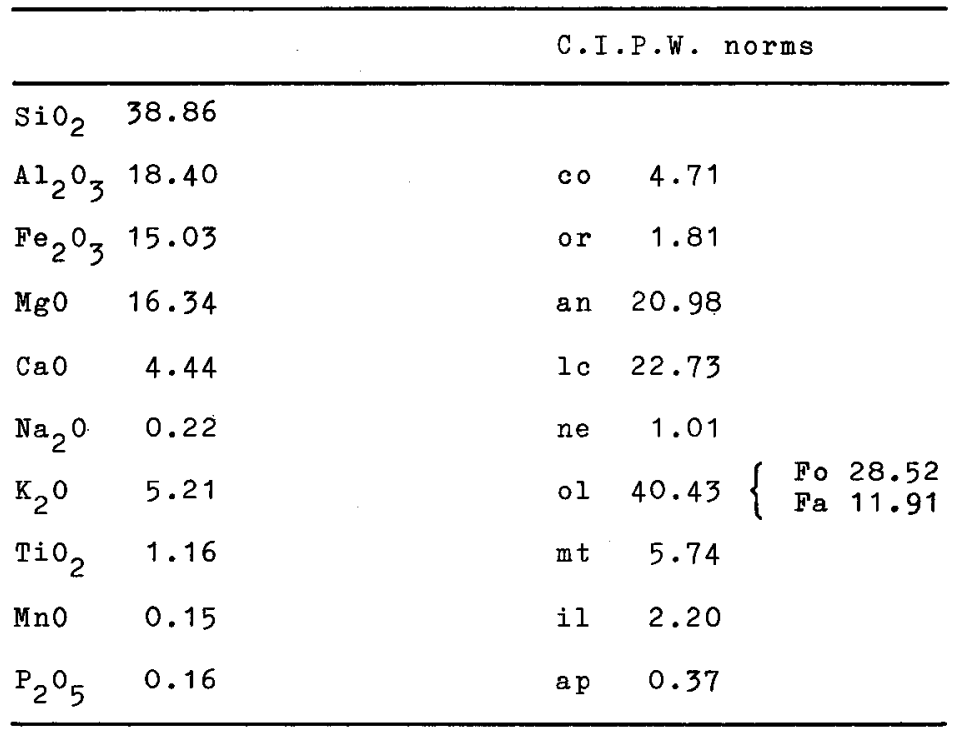

$\begin{aligned} \text { Analyst: } & \text { C.R.L. Friend, XRF data reduced by method } \\ & \text { of Brown et al. (1973) }\end{aligned}$

\section{Petrology}

The schists are medium to coarse-grained and fall into two main mineralogical groups (Table 13) based on the amphiboles they contain. They are then further subdivided on their additional mafic minerals.

Anthophyllite and cummingtonite are the amphiboles present (co-existing in 129766) and account for the pale colours of the rocks. Anthophyllite is weakly pleochroic pale brown, pale yellow-colourless, and is present either as radiating, acicular or columnar masses which may exceed $5 \mathrm{~cm}$ in length (fig. 41), or as tabular laths randomly scattered subparallel to the foliation planes. The formation of the amphiboles appears to be subsequent to the foliation as they are found frequently growing through it. Cummingtonite shows similar textures but is never so large and always occurs as thin, delicate needles. It is colourless and shows very common polysynthetic twinning.

Cordierite and staurolite are only found in those rocks containing anthophyllite (Table 13), and form a subdivision of these in three parts, one where the two minerals co-exist and two where each occurs singly. Staurolite occurs as scattered grains, sometimes showing euhedral faces, or as granular masses (fig. 42). Pleochroism in varying shades of yellow is marked. Cordierite is colourless to greyish (fig. 42), frequently poikiloblastic with distinctive pleochroic yellow halos around included zircon grains. In some specimens, a myrmekitic intergrowth with quartz is found (Lal \& Moorhouse, 1969). In twelve sections containing cordierite, no twinning has been found. Where cordierite and staurolite co- 


\begin{tabular}{|c|c|c|c|c|c|c|c|c|c|c|c|c|c|c|c|c|c|c|c|c|c|c|}
\hline GGU No. & $\begin{array}{l}\$ \\
+ \\
+4 \\
0 \\
0 \\
-1 \\
0 \\
0 \\
0 \\
0\end{array}$ & 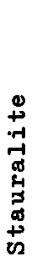 & 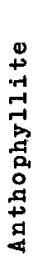 & $\begin{array}{l}0 \\
+ \\
+ \\
+ \\
0 \\
\stackrel{+}{m}\end{array}$ & 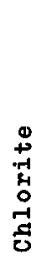 & 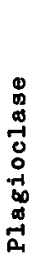 & 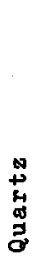 & 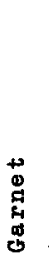 & 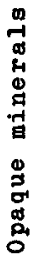 & 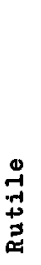 & 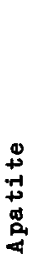 & GGU No. & 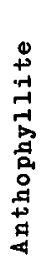 & 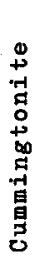 & 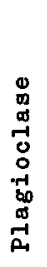 & 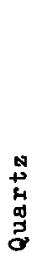 & 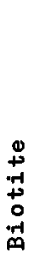 & 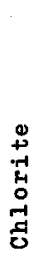 & $\underset{⿱ 中}{\Phi}$ & 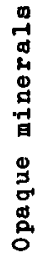 & 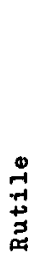 & $\begin{array}{l}\stackrel{\$}{+} \\
+ \\
+ \\
+ \\
0 \\
0\end{array}$ \\
\hline 119877 & $x$ & $\mathbf{x}$ & $\mathbf{x}$ & $\mathbf{x}$ & $\mathbf{x}$ & $x$ & $\mathbf{x}$ & & & $x$ & $\mathbf{x}$ & 119881 & $\mathbf{x}$ & & $\mathbf{x}$ & & $\mathbf{x}$ & $\mathbf{x}$ & & & $\mathbf{x}$ & \\
\hline $119878(3)$ & $x$ & $\mathbf{x}$ & $\mathbf{x}$ & $x$ & & $\mathbf{x}$ & $\mathbf{x}$ & $\mathbf{x}$ & $x$ & $\mathbf{x}$ & $\mathbf{x}$ & 119882 & $x$ & & $x$ & & $x$ & $x$ & & & & $x$ \\
\hline $128510(3)$ & $x$ & $\mathbf{x}$ & $\mathbf{x}$ & $x$ & $x$ & $\mathbf{x}$ & $\mathbf{x}$ & & $\mathbf{x}$ & & & $129579(2)$ & $\mathbf{x}$ & & $\mathbf{x}$ & $\mathbf{x}$ & $\mathbf{x}$ & $x$ & & $\mathbf{x}$ & & $\mathbf{x}$ \\
\hline 129732 & $\mathbf{x}$ & $\mathbf{x}$ & $x$ & $x$ & $\mathbf{x}$ & $\mathbf{x}$ & $\mathbf{x}$ & & $\mathbf{x}$ & $\mathbf{x}$ & & 129597 & $\mathbf{x}$ & & $\mathbf{x}$ & $\mathbf{x}$ & $\mathbf{x}$ & $\mathbf{x}$ & & & & \\
\hline 129768 & $\bar{x}$ & $\mathbf{x}$ & $\mathbf{x}$ & $\mathbf{x}$ & $\mathbf{x}$ & $\mathbf{x}$ & $\mathbf{x}$ & & & & $\mathbf{x}$ & 129733 & $\mathbf{X}$ & & $x$ & $x$ & $\mathbf{I}$ & & & $\mathbf{x}$ & & \\
\hline 129769 & $\mathbf{x}$ & $\mathbf{x}$ & $\mathbf{x}$ & $x$ & & $\mathbf{x}$ & $x$ & & $\mathbf{x}$ & & $x$ & $129775(4)$ & $\mathbf{x}$ & & $\mathbf{x}$ & $\mathbf{x}$ & $\mathbf{x}$ & $\mathbf{x}$ & & & & \\
\hline $\begin{array}{l}119890 \\
129763\end{array}$ & & $\mathbf{x}$ & $x$ & & & $\mathbf{x}$ & $x$ & & $\mathbf{x}$ & & $x$ & $\begin{array}{l}129767(2) \\
129924(2)\end{array}$ & $\begin{array}{l}x \\
x\end{array}$ & & $\mathbf{x}$ & & $x$ & $x$ & & $x$ & $x$ & $x$ \\
\hline 129103 & & $\mathbf{x}$ & $\mathbf{z}$ & $\bar{x}$ & & $\bar{x}$ & $\mathbf{x}$ & & $\mathbf{x}$ & & $\mathbf{x}$ & $129765 / 2$ & $x$ & & $x$ & $x$ & $\mathbf{x}$ & $x$ & & $x$ & & $x$ \\
\hline 129707 & $x$ & & $\mathbf{x}$ & $\mathbf{x}$ & $x$ & $\mathbf{x}$ & $x$ & & $\mathbf{x}$ & 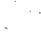 & $x$ & $129765 / 3$ & $\mathbf{x}$ & & $\mathbf{x}$ & $x$ & $\mathbf{x}$ & $\mathbf{x}$ & & & & \\
\hline 129774 & $x$ & & $x$ & $x$ & & $\mathbf{x}$ & $\mathbf{x}$ & & $\mathbf{x}$ & $\mathbf{x}$ & $\mathbf{x}$ & $129765 / 4$ & $\mathbf{x}$ & & $x$ & $\mathbf{x}$ & $\mathbf{x}$ & $\mathbf{x}$ & & & $\mathbf{x}$ & \\
\hline 129773 & $\mathbf{x}$ & & $\mathbf{x}$ & $x$ & $\mathbf{x}$ & $x$ & $\mathbf{x}$ & & $x$ & $\mathbf{x}$ & $x$ & $129961(4)$ & $\mathbf{x}$ & & $x$ & $\mathbf{x}$ & $\mathbf{x}$ & $\mathbf{x}$ & & $\mathbf{x}$ & $\mathbf{x}$ & \\
\hline 129963 & $x$ & & $x$ & & $x$ & $\mathbf{x}$ & $\mathbf{x}$ & & & $\mathbf{x}$ & $\mathbf{x}$ & 129777 & $x$ & & $\mathbf{x}$ & $\mathbf{x}$ & $x$ & & $x$ & $\mathbf{x}$ & & $\mathbf{x}$ \\
\hline . & & & & & & & & & & & & $129928(2)$ & $\mathbf{x}$ & & $x$ & $\mathbf{x}$ & $\mathbf{x}$ & $\mathbf{x}$ & $\mathbf{x}$ & & $\mathbf{x}$ & $\mathbf{x}$ \\
\hline Number of & $\mathbf{s} 11$ & des & in & n & pare & nt & hes & ses & & & & $\begin{array}{l}119880 \\
129701(3)\end{array}$ & & $\begin{array}{l}\mathbf{x} \\
\mathbf{x}\end{array}$ & $\begin{array}{l}\mathbf{x} \\
\mathbf{x}\end{array}$ & $\mathbf{x}$ & & & & $\mathbf{x}$ & $\mathbf{x}$ & $\mathbf{x}$ \\
\hline & & & & & & & & & & & & 129702 & & $\mathbf{x}$ & $\mathbf{x}$ & $\mathbf{x}$ & $\mathbf{x}$ & & & $\mathbf{x}$ & & $\mathbf{x}$ \\
\hline & & & & & & & & & & & & 129729 & & $x$ & $\mathbf{x}$ & & & & & $\mathbf{x}$ & & $\mathbf{x}$ \\
\hline$\cdot$ & & & & & & & & & & & & $129594(2)$ & & $x$ & $\mathbf{x}$ & $\mathbf{x}$ & & & & $x$ & & $x$ \\
\hline & & & & & & & & & & & & 129772 & & $\mathbf{x}$ & $\mathbf{x}$ & $\mathbf{x}$ & & & & $\mathbf{x}$ & $x$ & \\
\hline & & & & & & & & & & & & 129775 & & $\mathbf{x}$ & $\mathbf{x}$ & & & & & $\mathbf{x}$ & & \\
\hline & & & & & & & & & & & & $129963(3)$ & & $x$ & $\mathbf{x}$ & $\mathbf{x}$ & & & & & $x$ & \\
\hline & & & & & & & & & & & & 129926 & & $\mathbf{x}$ & $\mathbf{x}$ & $\mathbf{x}$ & & & & & $\mathbf{x}$ & \\
\hline & & & & & & & & & & & & 129766 & $\mathbf{x}$ & $x$ & $\mathbf{x}$ & & $\mathbf{x}$ & & & & & \\
\hline
\end{tabular}

exist they often exhibit a coronite relationship (fig. 42). Cordierite is frequently adjacent to a staurolite grain, or because of its poikiloblastic nature, surrounds one. On close inspection it is often found that whenever staurolite and cordierite are in contact, a narrow rim of cordierite surrounds the staurolite. Along this contact no visible reaction has taken place, the relationship appearing to be of simple overgrowth by the cordierite. This may, therefore, be entirely due to changing temperature and pressure conditions, since the two minerals are of approximately the same composition (Richardson, 1968). Biotite with alteration to chlorite is common. Plagioclase of oligoclase to andesine composition is present, and in cummingtonite-bearing rocks is the only felsic mineral (Table 13). Garnet appears only in rocks containing anthophyllite and is always highly poikiloblastic display- 


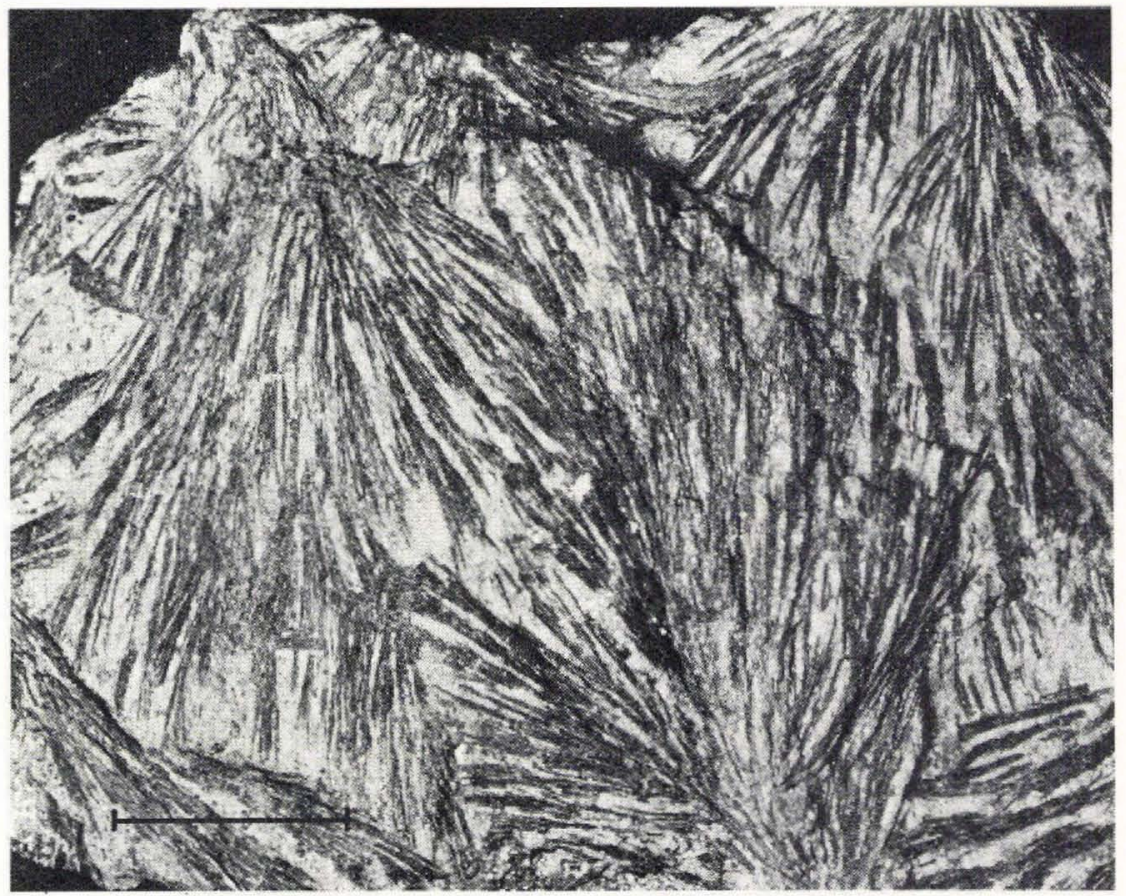

Fig. 41. GGU 119890 showing development of garbenschiefer texture in anthophyllite. Scale $2 \mathrm{~cm}$.

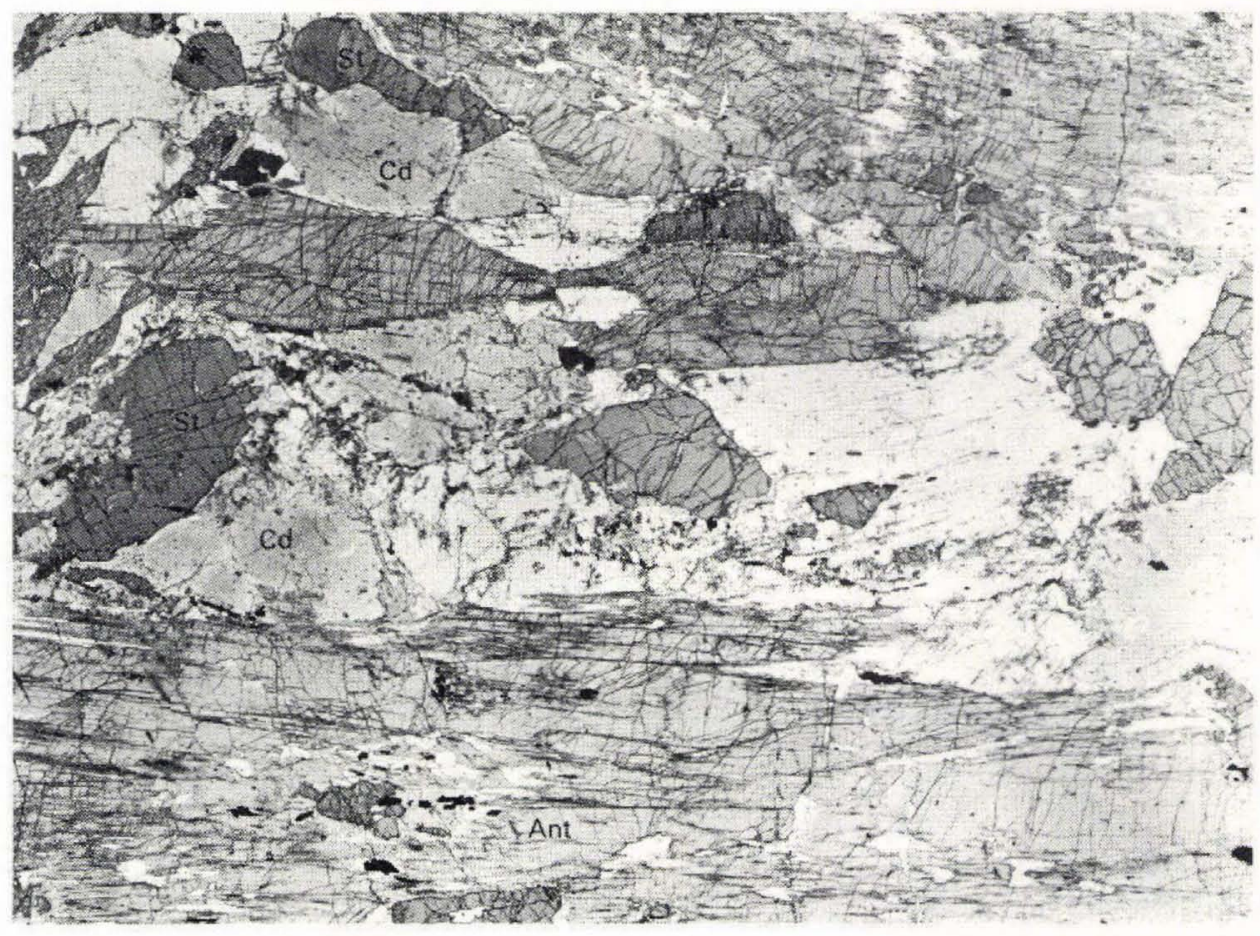

Fig. 42. Photomicrograph of co-existing cordierite and staurolite with anthophyllite. GGU 128510/a. St. Staurolite, Cd Cordierite, Ant Anthophyllite. $\times 2$. 
ing a snowball effect in the non-opaque and transparent inclusions. Apatite, zircon and rutile are accessories. Zircon is common in the cordierite-bearing rocks, where individual crystals can attain $0.3 \mathrm{~mm}$ in length.

\section{Metamorphic facies}

The minerals anthophyllite, staurolite, cordierite and garnet place the assemblages towards the top of the amphibolite facies (Turner, 1968). It is difficult to understand without microprobe work how cordierite could form when competing for $\mathrm{Mg}, \mathrm{Fe}$, and $\mathrm{Al}$ against staurolite, biotite, anthophyllite, and in one case (119878) garnet. Knowing the $\mathrm{Mg}-\mathrm{Fe}$ ratios, the experimental work on cordierite (Schreyer \& Yoder, 1964), cordierite-garnet stabilities (Hensen, 1972; Wiesbrod, $1973 \mathrm{a}, \mathrm{b}$ ), anthophyllite (Greenwood, 1963), and staurolite (Richardson, 1968), would allow the conditions under which these mineral associations formed to be accurately determined.

\section{References}

Andersen, L. S. \& Friend, C. R. L. 1973: Structure of the Ravns Storø amphibolite belt in the Fiskenæsset region. Rapp. Grønlands geol. Unders. 51, 37-40.

Brown, G. C., Hughes, D. J. \& Esson, J. 1973: New X. R. F. data retrieval techniques and their application to U.S.G.S. Standard rocks. Chem. Geol. 11, 223-229.

Dawes, P. R. 1970: Grønlandske granater som smykkesten. Grønland 1970, 113-119.

Giesecke, K. L. 1910: Karl Ludwig Gieseckes mineralogisches Reisejournal uber Grönland, 1806-1813. Meddr Grønland 35, 1-478.

Greenwood, H. J. 1963: The synthesis and stability of anthophyllite. J. Petrol. 4, 317-451.

Hensen, B. J. 1972: Cordierite-garnet equilibrium as a function of pressure and temperature and ironmagnesium ratio. Carnegie. Inst. Wash. Year Book 71, 418-421.

Lal, R. K. \& Moorhouse, W. W. 1969: Cordierite-gedrite rocks and associated gneisses of Fishtail Lake, Harcourt Township, Ontario. Can. Earth Sci. 6, 145-165.

Richardson, S. W. 1968: Staurolite stability in a part of the system Fe-Al-Si-O-H.J. Petrol. 9, 467-488. Spry, A. 1969: Metamorphic textures. Oxford: Pergamon Press.

Schreyer, W. \& Yoder, H. S. 1964: The system of Mg-cordierite- $\mathrm{H}_{2} \mathrm{O}$ and related rocks. Neues $J b$. Miner. Abh. 101, 271-342.

Turner, F. J. 1968: Metamorphic petrology. New York: McGraw Hill.

Wiesbrod, A. 1973a: Cordierite-garnet equilibrium in the system Fe-Mn-Al-Si-O-H. Carnegie Inst. Wash. Year Book 72, 515-518.

Wiesbrod, A. 1973b: Refinement of the equilibrium conditions of the reaction Fe-cordierite almandine $+\mathrm{Qtz}+$ Sillimanite $\left(+\mathrm{H}_{2} \mathrm{O}\right)$. Carnegie Inst. Wash. Year Book 72, 518-521. 